\title{
Direct access echocardiography to a district general hospital: are patients being screened appropriately?
}

\author{
Authors: Matthew Burton, Sheila Jen and Allan Harkness
}

\begin{abstract}
Aims
To identify the reasons for direct access echocardiography, to identify whether N-terminal pro-brain natriuretic peptide (NT pro-BNP) is used to target scanning effectively and to identify whether patients would otherwise have been referred to a cardiology clinic.
\end{abstract}

\section{Methods}

Two investigators studied all the echocardiograms done as a result of GP referrals for all reasons in a 1 month period retrospectively.

They completed a standardised audit tool looking at: the reason for referral; whether NT pro-BNP was measured and the value; documented history of previous myocardial infarction; the echocardiogram result; the time between referral and echocardiography; and whether, without direct access echocardiography, the GP would have referred the patient to a cardiology clinic.

\section{Results}

75 direct access echocardiograms were done in November 2013. 40 were referred for suspected heart failure (53\%) and 26 for murmurs $(35 \%)$.

22 of those referred for suspected heart failure (55\%) had NT pro-BNP levels measured prior to referral. It was positive in 15 patients $(68 \%)$.

Seven $(18 \%)$ patients had moderately or severely impaired left ventricular ejection fraction (LVEF $<45 \%)$. Four $(26 \%)$ of the patients with a positive NT pro-BNP had LVEF $<45 \%$; three $(15 \%)$ of the patients with no testing done had LVEF $<45 \%$. It is noted that seven patients with normal NT pro-BNP were still referred for echocardiography - none were identified to have LVEF impairment.

The average wait for echocardiography was 21 days.

Of the 75 referrals for echocardiography, 38 of the GPs would have otherwise referred to clinic versus four who would have treated; the remainder did not answer.

\section{Conclusions}

Most referrals are for clinically suspected heart failure and result in a normal echo.

Authors: Colchester Hospital University Foundation Trust, Colchester, UK
In referrals for suspected heart failure, there is a wellrecognised role of NT pro-BNP levels for identifying patients at higher risk. This is not being well utilised at present by our local primary care in screening their referrals. Better use of screening would reduce service demand without affecting patient care.

Direct referral echocardiography has a role in reducing potentially unneeded clinic appointments. Care has to be taken that appropriate patients are referred and that unnecessary investigations are not being done.

Consideration is being given to make positive NT pro-BNP an absolute prerequisite prior to direct access echocardiography for suspected heart failure.

\section{Conflict of interest statement}

The authors have no conflict of interest. 